\title{
ORIGINAL ARTICLE Reduced habituation of auditory evoked potentials indicate cortical hyper-excitability in Fragile X Syndrome
}

\author{
LE Ethridge ${ }^{1,2}$, SP White ${ }^{3}$, MW Mosconi ${ }^{3,4,5}, \mathrm{~J} \mathrm{Wang}^{3}$, MJ Byerly ${ }^{3}$ and JA Sweeney S $^{3,4}$
}

Sensory hypersensitivities are common, clinically distressing features of Fragile X Syndrome (FXS). Preclinical evidence suggests this abnormality may result from synaptic hyper-excitability in sensory systems. This model predicts reduced sensory habituation to repeated stimulus presentation. Fourteen adolescents and adults with FXS and 15 age-matched controls participated in a modified auditory gating task using trains of 4 identical tones during dense array electroencephalography (EEG). Event-related potential and single trial time-frequency analyses revealed decreased habituation of the N1 event-related potential response in FXS, and increased gamma power coupled with decreases in gamma phase-locking during the early-stimulus registration period. EEG abnormalities in FXS were associated with parent reports of heightened sensory sensitivities and social communication deficits. Reduced habituation and altered gamma power and phase-locking to auditory cues demonstrated here in FXS patients parallels preclinical findings with Fmr1 KO mice. Thus, the EEG abnormalities seen in FXS patients support the model of neocortical hyperexcitability in FXS, and may provide useful translational biomarkers for evaluating novel treatment strategies targeting its neural substrate.

Translational Psychiatry (2016) 6, e787; doi:10.1038/tp.2016.48; published online 19 April 2016

\section{INTRODUCTION}

Fragile $\mathrm{X}$ Syndrome (FXS) is a neurodevelopmental disorder associated with intellectual disability, facial dysmorphology and social deficits such as extreme shyness and anxiety. ${ }^{1}$ Auditory hypersensitivity is particularly common. ${ }^{2-4}$ While animal models for sensory hypersensitivity have been developed, little is known about neural underpinnings of this abnormality in FXS patients.

Animal models of FXS point to neuronal network hyperexcitability as a possible cause of sensory hypersensitivity. Local circuit hyper-excitability in Fmr1 knockout mouse models of FXS has been demonstrated, including prolonged 'UP' states in the gamma frequency range, decreased glutamatergic drive on fastspiking GABAergic inhibitory neurons in sensory cortex ${ }^{5}$ and heightened neurophysiological response to auditory stimuli. ${ }^{6}$ Inhibitory circuit deficits in these mouse models have been linked to abnormalities in both fast-spiking parvalbumin-expressing inhibitory interneurons $s^{5,7}$ and somatostatin-expressing lowthreshold-spiking inhibitory interneurons; ${ }^{8}$ these alterations may contribute directly to local circuit disorganization and hyperexcitability. Disorganized and hyper-excitable networks can have increased cortical 'noise' or desynchronous firing in the gamma frequency range that reduces stimulus selectivity and lowers the impact of local network output on postsynaptic circuits. ${ }^{9}$

Electroencephalography (EEG) can be used to characterize auditory processing abnormalities and cortical hyper-excitability in FXS. To date, few EEG studies in FXS have been conducted, especially studies of evoked sensory responses. ${ }^{10}$ The most common finding in FXS EEG studies has been enhanced early- sensory (N1) event-related potential (ERP) responses to auditory stimuli, which is consistent with neocortical hyper-excitability models of FXS. ${ }^{11-13}$

One approach for using EEG to assess sensory hypersensitivity is to utilize gating-type paradigms to determine whether there is a reduced $\mathrm{N} 1$ response to repeated stimulus presentations, reflecting reduced neural habituation in sensory cortex. The mechanism for sensory or short-term N1 habituation is believed to be related to refractoriness of cell populations involved in basic sensory processing systems rather than more complex cognitive processes exerting top-down control of sensory cortex. ${ }^{14,15}$ In typically developing subjects, reduced N1 suppression during sensory gating tasks have been associated with increased attention to irrelevant sounds ${ }^{16}$ and sensory avoidance on the Adolescent and Adult Sensory Profile, ${ }^{17}$ suggesting a link between N1 habituation and auditory hypersensitivity.

There is preliminary EEG evidence that individuals with FXS do not habituate to repeated sounds. ${ }^{1,18}$ However, previous habituation studies in FXS utilized an auditory oddball paradigm, a task in which rare novel (oddball) tones are presented in the context of a majority of standard (repeated) tones. Adding this probe of expectancy effects adds cognitive complexity and variable attentional influences to the assessment of basic auditory processing. ${ }^{12,16,19-21}$ Second, stimuli in prior studies were separated by relatively long inter-stimulus intervals, which can diminish habituation effects, as refractory circuits significantly complete their recovery after intervals longer than $500 \mathrm{~ms}^{22,23}$ When tones are presented in sequence with a shortened interstimulus interval and without novel distracters, cognitive factors

\footnotetext{
${ }^{1}$ Department of Pediatrics, Section on Developmental and Behavioral Pediatrics, University of Oklahoma Health Sciences Center, Oklahoma City, OK, USA; ${ }^{2}$ Department of Psychology, University of Oklahoma, Norman, OK, USA; ${ }^{3}$ Department of Psychiatry, Center for Autism and Developmental Disabilities, University of Texas Southwestern Medical Center, Dallas, TX, USA; ${ }^{4}$ Department of Pediatrics, University of Texas Southwestern Medical Center, Dallas, TX, USA and ${ }^{5}$ Departments of Applied Behavioral Science and Psychology, Schiefelbusch Institute for Life Span Studies and Clinical Child Psychology Program, University of Kansas, Lawrence, KS, USA. Correspondence: Dr LE Ethridge, Department of Pediatrics, Section on Developmental and Behavioral Pediatrics, University of Oklahoma Health Sciences Center, 1100 North East $13^{\text {th }}$ Street, Nicholson Tower, Suite 4900, Oklahoma City, OK 73104, USA.
}

E-mail: Lauren-Ethridge@ouhsc.edu

Received 16 October 2015; revised 11 February 2016; accepted 15 February 2016 
are limited and habituation effects seen in N1 reduction to repeated tones are increased. Limiting cognitive factors is particularly important when assessing sensory processing in groups with cognitive deficits such as FXS, where group differences in cognitive processing could obscure results. Thus, habituation might be better assessed using shorter inter-stimulus intervals ( $500 \mathrm{~ms} \mathrm{ISI)}$ in a passive listening paradigm.

Time-frequency analyses have revealed a relationship between increased power in the gamma frequency range and N1 amplitude abnormalities during sensory gating for other disorders with habituation deficits. These have been related to regulation of inhibitory interneuron circuits in sensory cortex, which act to bind stimulus features. ${ }^{24}$ Time-frequency analyses of evoked EEG data to our knowledge have not been conducted in FXS, but may be useful to investigate neural correlates of local circuit hyperexcitability, particularly in the gamma range where deficits have been observed in Fmrl mice and proposed to underlie sensory processing abnormalities. ${ }^{5}$

The present study investigated ERP and time-frequency components of short-term habituation to repeated tones in auditory cortex in individuals with FXS and healthy matched controls. Gamma activity was investigated to determine if there were similar increases in power in this frequency band in response to tones as has been reported in somatosensory cortex in Fmr1 mouse slice preparations, ${ }^{5}$ and if alterations in gamma activity were related to abnormalities in $\mathrm{N} 1$ habituation deficits as might be expected due to the role of gamma oscillations in maintenance of synchronous population activity in local cortical circuits. Electrophysiological abnormalities were correlated with parental reports of sensory and behavioral deficits in FXS patients to evaluate their relationship to sensory hypersensitivities and other behavioral problems associated with FXS.

\section{MATERIALS AND METHODS}

\section{Participants}

Fourteen adolescents and adults with full mutation FXS (mean age $=28.5$, s.d $=11.7$; age range 14-57; 3 females) and 15 age-matched controls (mean age $=28.9, \mathrm{~s} . \mathrm{d}=10.2$; age range $16-55 ; 5$ females) participated in the study. Previous studies support this sample size as sufficient to detect group differences. ${ }^{11-13,18}$ Healthy controls had no known prior diagnosis or treatment for psychiatric illness. Exclusion criteria included history of seizures and current use of anticonvulsant medications including benzodiazepines, or novel potential treatments for FXS (minocycline). Four patients were receiving atypical antipsychotics, and three antidepressants, all on a stable dose for at least 4 weeks. Medicated patients did not differ on primary EEG measures.

Clinical questionnaires including the Adolescent and Adult Sensory Profile, ${ }^{25}$ Social and Communication Questionnaire (SCQ), Achenbach Adult and Child Behavior Checklists (ABCL), and Aberrant Behavior Checklist $(A B C)$ were completed for FXS participants by their primary caregiver. Intelligence quotient (IQ) of FXS participants was assessed with the Stanford-Binet Intelligence Scale 5 th $\mathrm{Edn}^{26}$ which characterizes intellectual ability across a broad ability range. IQ of controls was estimated using the briefer Wechsler Abbreviated Scale of Intelligence (WASI). ${ }^{27}$ All participants provided written informed consent (caregiver with assent or individual consent as appropriate) prior to participation, as approved by the UT Southwestern Institutional Review Board.

\section{Procedure}

Stimuli consisted of 150 trials; each trial consisted of a train of four $1000 \mathrm{~Hz}$ tones (50-ms duration). Tones within each train were separated by a $500-$ ms inter-stimulus interval; trials were separated by a $4000-\mathrm{ms}$ interval. Tones were delivered at $65 \mathrm{db}$ through headphones, while participants underwent dense array EEG. Participants watched a silent movie to facilitate compliance.

\section{ERP recording}

EEG was continuously recorded and digitized at $512 \mathrm{~Hz}$, with a 5th-order Bessel anti-aliasing filter at $200 \mathrm{~Hz}$, using a 128 channel BioSemi ActiveTwo system (BioSemi, Amsterdam, Netherlands) with sensors placed according to the International $10 / 10$ system. ${ }^{28}$ All sensors were referenced to a monopolar reference feedback loop connecting a driven passive sensor and a common mode sense active sensor, both located on posterior scalp.

\section{EEG analysis}

Raw data were visually inspected offline. Bad sensors were interpolated using spherical spline interpolation implemented in BESA 6.0 (MEGIS Software, Grafelfing, Germany). Data were digitally filtered from 0.5 to $55 \mathrm{~Hz}$ ( $6 \mathrm{db}$ and $12 \mathrm{db}$ per octave rolloff, respectively; zero-phase) and re-referenced to average reference. Eye movement, cardiac and muscle movement (EMG) artifacts were removed blind to group using independent components analysis (ICA) implemented in EEGLAB 11 (ref. 29) in Matlab (The Mathworks, Natick, MA, USA). Data were epoched into $3000 \mathrm{~ms}$ trials ( -500 to $2500 \mathrm{~ms}$ ), averaged across trials and baselinecorrected using the 500-ms period prior to the first tone in each trial. Any trial with post-ICA amplitude exceeding $100 \mu \mathrm{V}$ was considered residual artifact and removed prior to averaging. All subjects retained at least $75 \%$ of trials in the final analyses (FXS $M=137.6$, s.d. $=8.8$; Control $M=144.3$, s.d. $=4.3$ ).

To take advantage of the dense electrode array and integrate data from every sensor, spatial principal components analysis (PCA) was implemented on the grand average ERP. ${ }^{30,31}$ Component weights were multiplied by each subject's average data, summed across sensors and divided by the sum of the component weights, reducing waveforms from one for each sensor to one waveform per component with a defined spatial distribution across the scalp.

Spectral analyses using Morlet wavelets with $1 \mathrm{~Hz}$ resolution were conducted on epoched single trial data weighted by the average PCA component topographies. To balance time resolution in lower frequencies with stability in higher frequencies, ${ }^{32}$ wavelets were calculated using a linearly increasing cycle length from 1 cycle at the lowest frequency $(2 \mathrm{~Hz})$ to 10 cycles at the highest $(55 \mathrm{~Hz})$. Single trial power (STP) and inter-trial coherence (ITC) measures were obtained to evaluate amplitude of response at each frequency and how stable or phase-locked responses were across trials, respectively. ${ }^{29}$ STP and ITC values were averaged over trials for each individual and transformed into time-frequency plots downsampled to 300 time-bins. ${ }^{33}$ Group differences were calculated using two-sided $t$-tests at each point in the time-frequency matrix. In particular, based on mouse model studies ${ }^{5,7,8}$ we predicted that FXS participants would show non-stimulus-specific increases in gamma STP due to hyperexcitable or 'noisy' local cortical networks.

Control for multiple comparisons was achieved using time-frequency clustering techniques and Monte Carlo simulations. ${ }^{30,33}$ To maintain a family-wise a of 0.01 , a minimum of three sequential time-bins and three adjacent frequencies were required to be significant at a nominal threshold of $P<0.05$.

\section{Statistical analysis}

Amplitude and latency of the $\mathrm{N} 1$ component to each tone was measured for each individual at the peak, defined as the most negative-going waveform deflection between 50 and 150 ms post stimulus, and verified through visual inspection.

We limited the number of repetitions to 3 as prior habituation literature suggests that the majority of habituation effects are present by that time. ${ }^{34}$ The exponential decay function is not well-suited to datasets with such a limited number of time points. Therefore habituation of N1 amplitude across repeated tones in a trial was quantified as percent change from the first tone in each trial, with a focus on comparing fast decay (habituation to the first repeated tone) with asymptote (habituation to the fourth tone) to quantify habituation effects. Percent change across repetitions was analyzed using a repeated measures analysis of variance comparing repetition number in sequence (first, second or third) by group (FXS vs control). ${ }^{34}$ All repeated measures tests included Greenhouse-Geisser correction. Latency and amplitude were measured for the P2, defined as the largest positive-going peak between 150 and $250 \mathrm{~ms}$ post stimulus. The N2 component was less easily visualized in some participants, so it was quantified as the average amplitude over $30 \mathrm{~ms}$ centered on the N2 peak amplitude in the grand average and latency was not calculated. 
Clinical correlations were examined with $\mathrm{N} 1$ amplitude to the initial tone, average N2 amplitude across all four tones, percent change in N1 amplitude, gamma STP and all significant time-frequency clusters using Spearman's $\rho$. To examine whether such an abnormal increase in high frequency 'noise' was associated with abnormalities in habituation, correlations between gamma STP, gamma ITC, low frequency ITC, N1 amplitude and N1 percent change were conducted using Spearman's $\rho$. Clinical scales were prioritized based on their applicability to sensory and social abnormalities (Sensory Profile, SCQ). Clinical correlations are presented as exploratory, not corrected for multiple comparisons.

\section{RESULTS}

Clinical and demographic

FXS patients showed significantly lower full scale IQ than controls (Table 1). Most of the patients scored in the intellectually disabled range, with three patients scoring in the low-normal range; however, EEG and clinical values did not differ for these patients, so they were retained in the analyses. FXS showed significantly higher Sensory Profile and SCQ scores than controls (Table 1).

\section{Event-related potential}

Spatial PCA revealed a single spatial component representing $93.0 \%$ of the variance that was consistent with known auditory N1/P2/N2 topography (Figure 1). Subsequent analyses were performed on a virtual sensor created by weighting ERP or trialwise EEG data by the PCA component weights. There was no significant group difference in $\mathrm{N} 1$ amplitude to the initial stimulus, $t(27)=1.7, P=0.09$. In a repeated measures analysis of variance on percent change across repetitions, there was a main effect of group, $F(1,27)=6.9, P=0.014$, indicating that FXS showed less habituation of the N1 ERP than healthy controls (Figure 1). There was also a main effect of repetition number, $F(2,54)=4.7$, $P=0.016$. Subjects showed a significant increase in habituation between repetition 1 and $2, t(27)=2.54, P=0.17$, (mean percent change repetition $1=31 \%$, s.d. $=21 \%$; mean percent change repetition $2=38 \%$, s.d. $=18 \%$ ) but no difference in habituation between repetitions 2 and 3 , indicating asymptote of habituation by the fourth stimulus in the trial, consistent with findings in typically developing individuals. ${ }^{34,35}$ The repetition number by group interaction was not significant, indicating no difference in rate of habituation between groups. This result suggests that additional repetitions do not result in eventual normalization of N1 habituation by allowing FXS to 'catch up' with more gradual habituation of sensory responses. Gender was entered as a covariate but was not significant, so was removed from the final model. There was no group difference in N1 latency or P2 amplitude or latency for any of the four stimuli.

N2 amplitude did not show a significant habituation effect, so amplitude was collapsed over all four stimuli. There was a significant group difference in overall N2 amplitude, $t(27)=3.7$, $P=0.001$, with FXS participants showing significantly reduced N2 amplitude (mean $=0.19 \mu \mathrm{V}$, s.d. $=0.73$ ) relative to healthy controls (mean $=-0.88 \mu \mathrm{V}$, s.d. $=0.82$ ).

\section{Time-frequency analyses}

Because baseline STP differed between groups, with FXS showing increased baseline gamma power compared with controls $(t$ $(27)=3.36, \quad P=0.002$; Figure 2b), STP was analyzed both as absolute power with no baseline-correction to show overall power differences between groups and also using the prestimulus period from -220 to $-50 \mathrm{~ms}$ (chosen to avoid windowing effects) to baseline-correct STP for each individual to examine relative STP changes associated with stimulus-related neural activity. Point-by-point $t$-tests on time-frequency plots for ITC, STP and baseline-corrected STP (corrected for multiple comparisons) revealed 18 time-frequency clusters with significant differences between FXS and controls (Figure 2, Table 2). Cluster names are identified by stimulus number (initial stimulus or repetition 1, 2 or 3), ERP associated with the time period (if applicable) and frequency band. Time ranges are given relative

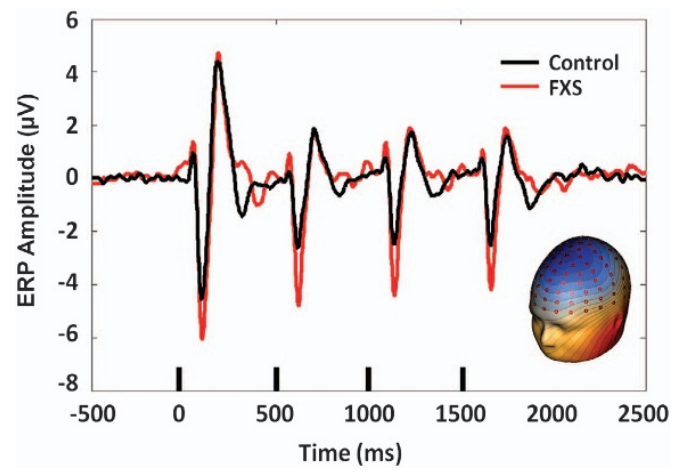

Figure 1. ERP grand average PCA-weighted virtual channel plot for FXS and matched controls, with inset PCA spatial component topography. Small black bars indicate presentation of the auditory stimulus. ERP, event-related potential; FXS, Fragile X Syndrome; PCA, principal components analysis.

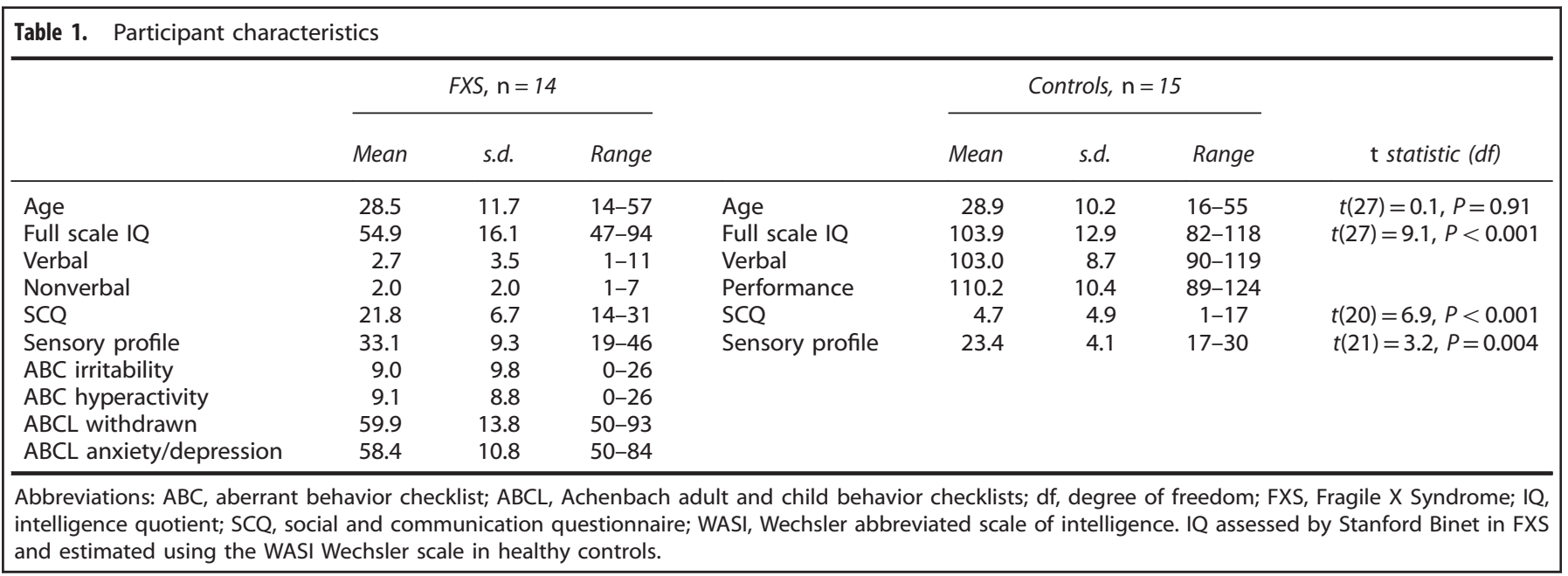




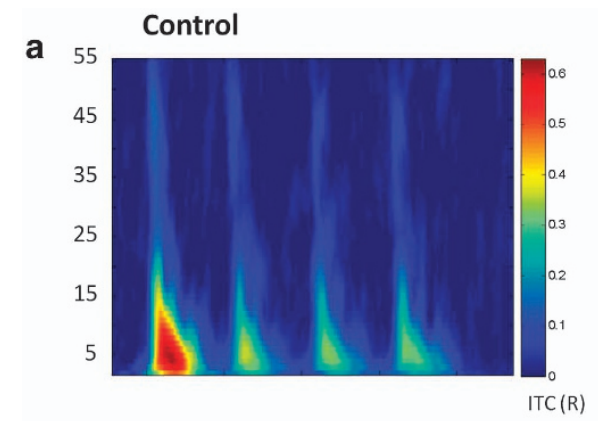

FXS

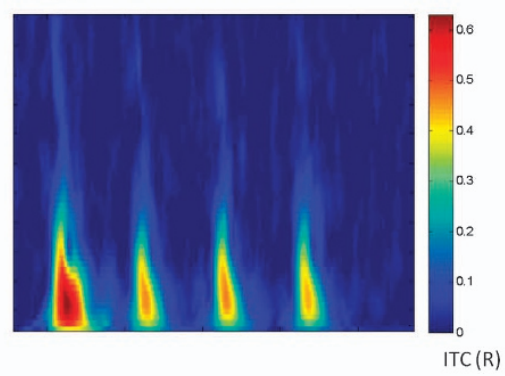

FXS - Control

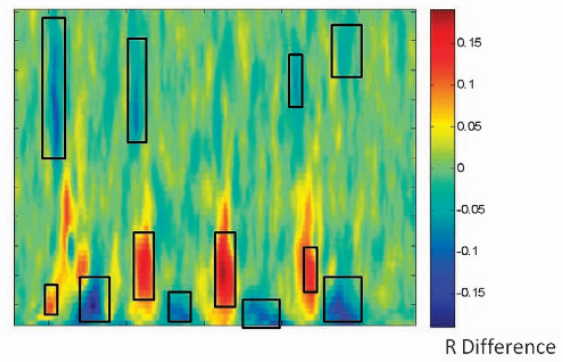

b
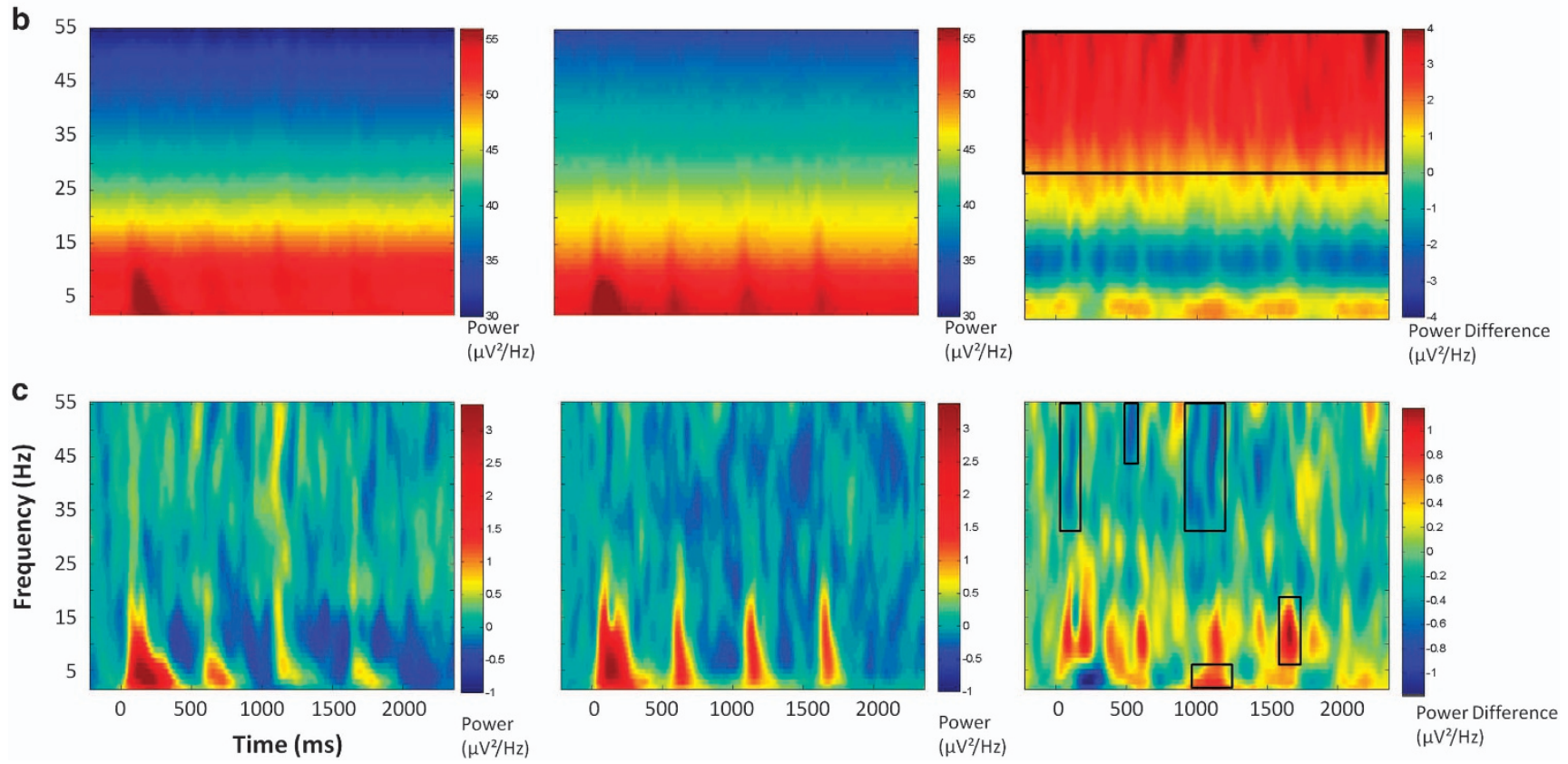

Figure 2. (a) ITC. (b) Single trial power. (c) Baseline-corrected single trial power. Black boxes in the difference maps indicate clusters with significant group differences. Warmer colors in the difference maps (right column) indicate higher phase-locking or higher power for FXS and cooler colors indicate higher values for healthy controls. FXS, Fragile X Syndrome; ITC, inter-trial coherence.

to initial stimulus onset, to provide equivalency with the $x$-axes in Figure 2. Cluster peaks were identified for highest $t$-values in group comparisons, not for peaks of activity. For absolute gamma STP, a peak statistic is reported, however, group differences were remarkably stable throughout the trial. In general, FXS patients showed significantly increased phase-locking in low frequencies during the $\mathrm{N} 1$ time period and decreased phase-locking during the N2 time period (Figure 2a), consistent with the increased amplitude of the N1 ERP and decreased amplitude of the N2 ERP. FXS showed decreased gamma phase-locking during the N1 time period (Figure 2a), and a general increase in absolute gamma power throughout the trial (Figure $2 b$ ).

Due to significant group differences in low frequency ITC clusters for each repetition, which paralleled N1 ERP findings, identical habituation analyses were performed for ITC using cluster means. Because a group difference cluster of similar size did not exist for low frequency ITC to the initial stimulus presentation, ITC data for each subject was averaged over a representative time period of $0-150 \mathrm{~ms}$ post stimulus using the same low frequency range in which group differences were found for the repetitions. Similar to the ERP results, FXS showed significantly less percent change in low frequency ITC relative to controls $F(1,27)=13$. 23, $P=0.001$. There was no effect of repetition number or group by repetition interaction.

\section{Clinical correlations}

Significant correlations with clinical measures in FXS participants are presented in Table 3. Increased N1 amplitude to the initial stimulus and reduced habituation were related to clinical measures of sensory and behavioral reactivity.

\section{Gamma power and ERPs}

For FXS, increased gamma STP above the elevated baseline to the initial stimulus was correlated with less habituation to the first repetition $(\rho=-0.61, P=0.02)$, and increased gamma STP to the first repetition was correlated with less habituation to the second repetition $(\rho=-0.57, P=0.03)$. Absolute increases in gamma STP throughout the trial were significantly correlated with decreases in gamma ITC to the initial stimulus $(\rho=-0.54, P=0.04)$, which in turn was correlated with increased N1 ERP to the same stimulus $(\rho=-0.59, P=0.03)$. Increased gamma power in FXS, both absolute increases relative to healthy controls and further increases above an already elevated baseline, can be thought of as an increase in background neural 'noise'. This increase in nonspecific high frequency neural activity contributed to a decreased ability to synchronize gamma frequency activity when necessary (during initial stimulus representation), which was associated with an overgeneralized excitatory response (increased N1 amplitude), as well as lowered ability to habituate to repetition. 
Table 2. Time-frequency clusters with significant group differences

\begin{tabular}{|c|c|c|c|c|c|}
\hline Cluster label & Time range & Peak time & Peak frequency & Statistic & Direction of group difference \\
\hline Initial stimulus pre-N1 delta/theta & -22 to $30 \mathrm{~ms}$ & $-4 \mathrm{~ms}$ & $5 \mathrm{~Hz}$ & $t(27)=3.37, P=0.002$ & $\mathrm{FXS}>\mathrm{CON}$ \\
\hline Repetition 1 N1 alpha/beta & 556 to $658 \mathrm{~ms}$ & $642 \mathrm{~ms}$ & $13 \mathrm{~Hz}$ & $t(27)=3.09, P=0.005$ & $\mathrm{FXS}>\mathrm{CON}$ \\
\hline Repetition $1 \mathrm{~N} 1$ gamma & 530 to $598 \mathrm{~ms}$ & $556 \mathrm{~ms}$ & $36 \mathrm{~Hz}$ & $t(27)=4.05, P<0.001$ & $\mathrm{CON}>\mathrm{FXS}$ \\
\hline Repetition $1 \mathrm{~N} 2$ delta/theta & 796 to $918 \mathrm{~ms}$ & $849 \mathrm{~ms}$ & $4 \mathrm{~Hz}$ & $t(27)=2.79, P=0.009$ & $\mathrm{CON}>\mathrm{FXS}$ \\
\hline Repetition 3 N1 alpha & 1660 to $1720 \mathrm{~ms}$ & $1685 \mathrm{~ms}$ & $11 \mathrm{~Hz}$ & $t(27)=3.02, P=0.005$ & $\mathrm{FXS}>\mathrm{CON}$ \\
\hline Repetition 3 N1 gamma & 1574 to $1616 \mathrm{~ms}$ & $1591 \mathrm{~ms}$ & $42 \mathrm{~Hz}$ & $t(27)=4.14, P<0.001$ & $\mathrm{CON}>\mathrm{FXS}$ \\
\hline Repetition 3 N2 delta/theta & 1806 to $2030 \mathrm{~ms}$ & $1867 \mathrm{~ms}$ & $5 \mathrm{~Hz}$ & $t(27)=3.91, P<0.011$ & $\mathrm{CON}>\mathrm{FXS}$ \\
\hline Repetition 3 N2 gamma & 1806 to $1910 \mathrm{~ms}$ & $1884 \mathrm{~ms}$ & $49 \mathrm{~Hz}$ & $t(27)=4.36, P<0.001$ & $\mathrm{CON}>\mathrm{FXS}$ \\
\hline \multicolumn{6}{|l|}{ Single trial power } \\
\hline Overall gamma & -220 to $2350 \mathrm{~ms}$ & $1798 \mathrm{~ms}$ & $39 \mathrm{~Hz}$ & $\mathrm{~F}(1,27)=10.1, P=0.004^{\mathrm{a}}$ & $\mathrm{FXS}>\mathrm{CON}$ \\
\hline Repetition 2 N1 gamma & 934 to $1150 \mathrm{~ms}$ & $961 \mathrm{~ms}$ & $37 \mathrm{~Hz}$ & $t(27)=3.27, P=0.003$ & $\mathrm{CON}>\mathrm{FXS}$ \\
\hline Repetition 3 N1 alpha/beta & 1600 to $1702 \mathrm{~ms}$ & $1668 \mathrm{~ms}$ & $13 \mathrm{~Hz}$ & $t(27)=2.93, P=0.007$ & $\mathrm{FXS}>\mathrm{CON}$ \\
\hline \multicolumn{6}{|c|}{$\begin{array}{l}\text { Abbreviations: ANOVA, analysis of variance; CON, control; ERP, event-related potential; FXS, Fragile X Syndrome; ITC, inter-trial coherence. Cluster names are } \\
\text { identified by stimulus number (Initial stimulus or repetition } 1,2 \text { or } 3 \text { ), ERP associated with the time period (if applicable) and frequency band. Time ranges are } \\
\text { given relative to initial stimulus onset, to provide equivalency with the } x \text {-axes in Figure } 2 \text {. Repetitions occurred every } 500 \text { ms. Cluster peaks are identified for } \\
\text { highest } t \text {-values, not for peaks of activity. For overall gamma single trial power, a peak statistic is reported, however, it should be noted that group differences } \\
\text { were remarkably stable throughout the time period. }{ }^{2} \text { Number of trials retained was significantly correlated with overall gamma single trial power in FXS, so } \\
\text { number of trials was included as a factor in an ANOVA for this group comparison only. Group differences remained significant. }\end{array}$} \\
\hline
\end{tabular}

Table 3. Significant clinical correlations

\begin{tabular}{|c|c|c|c|c|c|}
\hline \multirow[t]{2}{*}{ EEG measure } & \multicolumn{5}{|c|}{ Clinical scale } \\
\hline & $\begin{array}{l}\text { Sensory profile- } \\
\text { auditory }\end{array}$ & $A B C L$-withdrawn & $A B C$-hyperactivity & $A B C$-irritability & SCQ total score \\
\hline N1 amplitude-initial tone & $-0.67^{*}$ & - & - & - & - \\
\hline $\begin{array}{l}\text { Percent change N1 amplitude-asymptote (final } \\
\text { repetition) }\end{array}$ & $-0.65^{*}$ & $-0.81^{* *}$ & $0.76^{*}$ & - & - \\
\hline $\begin{array}{l}\text { Percent change N1 ITC-asymptote (final } \\
\text { repetition) }\end{array}$ & $0.59^{*}$ & $-0.89 * *$ & - & - & - \\
\hline Delta/theta ITC (N2 initial stimulus) & $-0.64^{*}$ & - & - & - & - \\
\hline Alpha/beta ITC (N1 first repetition) & $0.67^{*}$ & - & - & $0.69^{*}$ & - \\
\hline Alpha ITC (N1 second repetition) & $0.69 *$ & - & - & - & - \\
\hline Alpha ITC (N1 final repetition) & $0.67^{*}$ & $0.76^{*}$ & $0.77^{* *}$ & $0.74 *$ & - \\
\hline Baseline-corrected gamma STP (N1 initial stimulus) & - & $0.67^{*}$ & $0.70^{*}$ & $0.68^{*}$ & $0.89^{* *}$ \\
\hline Alpha/beta STP (N1 final repetition) & $0.61 *$ & - & - & - & - \\
\hline
\end{tabular}

Abbreviations: $A B C$, aberrant behavior checklist; $A B C L$, Achenbach adult and child behavior checklists; df, degree of freedom; EEG, electroencephalography; ITC, inter-trial coherence; NS, not significant; SCQ, social and communication questionnaire. Note: for amplitude correlations on negative ERP components N1 and N2 (first and fourth items in the first column), negative correlations indicate an increased amplitude of response correlated to increased scores on the clinical scale, while positive correlations indicate a decreased amplitude of response correlated to increased scores on the clinical scale. EEG measures with no significant correlations to clinical variables are not included. All correlations are Spearman's $\rho$. '-' indicates NS. ${ }^{*} P<0.05$. ${ }^{* *} P<0.01 .{ }^{* * *} P<0.001$.

To obtain an overall view of excitatory vs inhibitory abnormalities in FXS, all subjects were ranked from 1-29 in order from lowest to highest individual value for the primary variables indicating change in excitatory (gamma STP, N1 amplitude to initial stimulus) and inhibitory (gamma phase-locking during 'gamma spike' for initial stimulus and first repetition) activity, then composite variables were created by averaging these rankings in each category. Composite variables were plotted for FXS and control participants to characterize the relative imbalance in excitatory vs inhibitory activity (Figure 3 ). Controls show high inhibitory, organized activity and low background excitatory noise. In contrast, most FXS participants have both higher excitatory response and lower inhibitory activity.

\section{DISCUSSION}

Sensory processing abnormalities are common and clinically distressing features of FXS. Inability to habituate to sensory events and ongoing background noise may contribute to hypersensitivity to sounds and overstimulation. Behavioral and 


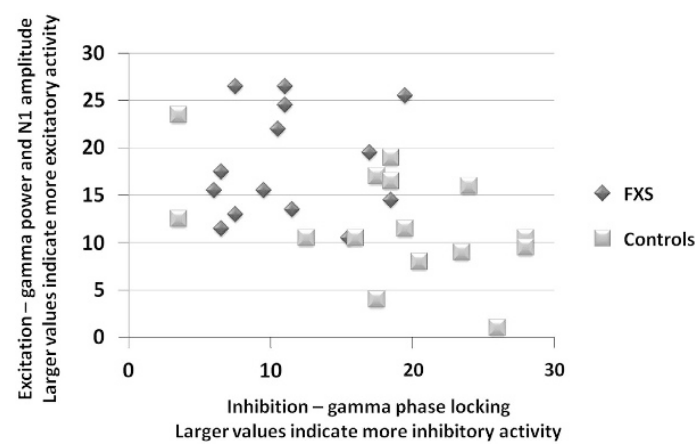

Figure 3. Relationship of excitatory (gamma single trial power, N1 amplitude to initial stimulus) and inhibitory (gamma phase-locking during 'gamma spike' for initial stimulus and first repetition) activity in response to trains of auditory stimuli. Units for both $x$ and $y$ axes represent averaged rank scores from lowest (1) to highest (29) for the composite variables. Controls show high inhibitory, organized activity and low background excitatory noise. In contrast, most FXS participants have both higher excitatory response and lower inhibitory activity. FXS, Fragile X Syndrome.

physiological findings have suggested deficits in short-term habituation in FXS, including reduced prepulse inhibition of the startle response ${ }^{36-38}$ and abnormalities in electrodermal activity to repetitive stimuli, ${ }^{39}$ however, neural correlates for these findings have not been elucidated. We believe the current study provides the first direct clinical evidence for a contribution of gamma band abnormalities, suggesting an imbalance of excitatory vs inhibitory activity, an effect found in mouse models of FXS, and the first evidence that these are clinically relevant by virtue of their relationship to parental reports of sensory sensitivities in FXS patients.

The current study also provides the first direct evidence of short-term local circuit-based habituation deficits in FXS patients. FXS patients showed a significant decrease in N1 suppression (both ERP amplitude and low frequency phase-locking) to repeated stimuli. This alteration was also associated with parental reports of auditory hypersensitivity and social problems in FXS participants. N1 habituation did not normalize across additional repetitions of the stimulus, suggesting a fundamental deficit in organization of local networks representing basic auditory stimulus properties. Although overall N1 amplitude has been the focus of previous work, ${ }^{11-13} \mathrm{~N} 1$ amplitude to the initial nonhabituated stimulus did not differ between groups (though there was a non-significant trend) and it was not significantly correlated with habituation $(\rho=0.15, P=0.61)$, suggesting potentially dissociable mechanisms between neural excitation (initial N1 amplitude) $)^{10,11}$ and inhibition (habituation). ${ }^{14}$

While N1 amplitude to the initial non-habituated stimulus did not differ between groups as in some previous studies, ${ }^{11,18}$ increased $\mathrm{N} 1$ amplitude and increased low frequency ITC during the $\mathrm{N} 1$ time period that contributes to formation of the N1 ERP, ${ }^{40}$ were correlated with increased abnormalities on the Sensory Profile, suggesting that individuals with high ERP amplitudes may have more severe sensory symptoms. Previous studies finding N1 differences used younger ${ }^{11,18}$ and/or smaller ${ }^{11}$ samples, and their level of sensory sensitivities is unknown. The current finding of decreased N2 amplitude in FXS is consistent with findings of some ${ }^{11}$ but not all prior studies. ${ }^{13}$ The long inter-stimulus interval single stimulus task employed by Knoth et al. ${ }^{13}$ may have decreased N2 amplitudes and their utility for detecting deficits. The N2 ERP is commonly associated with incorporation of frontal generators in stimulus identification and maintenance of auditory memory traces. ${ }^{41}$ Decreased amplitude of N2 was associated with increased scores on the SCQ, Sensory Profile and ABC Irritability component, suggesting a broad sensory and behavioral impact of this alteration.

Using time-frequency analyses of evoked EEG for the first time in FXS, the current study provides evidence for both increased gamma 'noise' (increased desynchronous high frequency firing) and decreases in ability to organize inhibitory networks to synchronize gamma band activity in the brain. Broad increases in excitation of gamma circuits without corresponding increases in synchronization of those circuits are consistent with presence of heightened neocortical excitability in FXS, and may represent a mechanism underlying sensory hypersensitivities in this disorder and the observed neural habituation deficit.

Higher ongoing or background gamma power in FXS relative to controls was not locked in time to stimulation. Individuals with elevated background gamma activity showed decreased ability to coherently organize and phase-lock gamma activity during the 'gamma spike' period, a short burst of phase-locked gamma activity that normally occurs during early-stimulus processing. ${ }^{42}$ Decreased phase-locking during the gamma spike was associated with greater N1 amplitudes, suggesting an overall increase in neural background noise that contributes to both hyperexcitability (increased N1) and disorganization (decreased ability to 'lock in' or synchronize gamma response to the stimulus). Transient increases in gamma power above the already elevated baseline in response to each stimulus were associated with poorer habituation response to the following stimulus, suggesting that this increase in power is not beneficial or compensatory, but rather reflects an over-recruitment of local networks, which generally oscillate in the gamma range. ${ }^{43}$

In a normally functioning local circuit, refractory properties of the network prevent the same circuit from activating as strongly to repeated stimuli presented in close succession, creating shortterm habituation. ${ }^{14}$ Less synchronized and more widely excitable local synaptic networks would mean that the same circuit of synapses may not be excited in the same way for each repetition, preventing proper functioning of the refractory system. Long-term depression, which weakens synaptic connections in a neural circuit, is enhanced in Fmr1 knockout mouse models of $\mathrm{FXS}^{44}$ weaker connections between neurons in a given local circuit coupled with increased firing of unrelated neurons may also account for that circuit's inability to reliably activate and thus habituate to a repeated stimulus. Importantly, these findings suggest not only a promising avenue for investigating brain dysfunction in FXS, but a promising translational strategy for integrating rodent and patient studies and developing mechanistically important biomarkers useful for advancing drug discovery.

Gamma hyper-excitability in Fmr1 knockout mice has been related to decreased excitatory drive on fast-spiking inhibitory interneurons, which is associated with decreased synchronization or phase-locking in the gamma range. ${ }^{5}$ Decreased activation of fast-spiking inhibitory interneurons that operate in the gamma range and drive the local neural oscillatory response could account for both local circuit hyper-excitability and reduced neural habituation. Reduced inhibitory drive onto pyramidal cells in auditory cortex from fast-spiking interneurons would lead to increased cell firing and less coherent organization and tuning to sensory stimuli. In the current study, increases in non-phaselocked gamma power were correlated with decreases in phaselocked gamma activity, suggesting a hyper-excitable but disorganized system consistent with these circuit dynamics.

The decrease in phase-locked gamma was also correlated with increased N1 amplitudes, suggesting a connection between local circuit hyper-excitability and increased recruitment of neural generators for sensory evoked potentials. Decreased habituation to a given repetition was associated with increased gamma power to the preceding stimulus, suggesting a direct relation between increased background high frequency 'noise' activating extraneous neural circuits during early-stimulus processing that could 
disrupt refractory-based circuit inhibition to the next repetition of that stimulus. Of note, N1 amplitudes were heterogeneous and suggest a subset of individuals with abnormally high N1, but habituation and gamma function were more consistently abnormal and associated with clinical measures.

Certain limitations in this study need to be considered. First, we cannot determine the degree to which our findings represent FXSspecific factors or alterations associated with developmental or intellectual disabilities. For example, there is evidence that individuals with Down syndrome may also show abnormal habituation to repetitive auditory stimuli, ${ }^{45,46}$ though individuals with Down syndrome generally show decreased or normal ongoing and evoked high beta and gamma power, ${ }^{47,48}$ which is opposite of that observed here in FXS. Second, FXS participants were taking various medications, and their potential impact on the data, while not apparent in statistical analyses, cannot be ruled out. Third, clinical correlations were performed on an exploratory basis only, and require validation via replication in additional samples. Last, although no gender differences were found, we had a limited number of FXS female participants.

To the best of our knowledge, this study presents the first comprehensive analysis of short-term auditory habituation in FXS. Our findings are consistent with a model that abnormalities in local circuit function leads to a hyper-excitability of sensory cortex that contributes to altered sensory experiences in FXS and other behavioral problems. Given the proposed linkage of circuit-based abnormalities in FXS with sensory processing abnormalities, reduced habituation and gamma abnormalities may represent promising candidate translational biomarkers for FXS. In FXS mice, minocycline treatment reduces audiogenic seizures, ${ }^{49}$ partially reduces aberrant $\mathrm{N} 1$ amplitudes and improves $\mathrm{N} 1$ habituation, ${ }^{18}$ suggesting that circuit dynamics associated with habituation may be good translational targets for drug development. Gamma abnormalities in particular may help track and explain hypothesized excitatory/inhibitory imbalances in FXS, and may contributed to lower seizure thresholds. Concurrent work with EEG in FXS mouse models ${ }^{50}$ will further elucidate molecular mechanisms underlying these electrophysiological alterations and their potential use as translational biomarkers for new targeted treatments for FXS. Such work might also lead to new treatment options for neural hyper-excitability and sensory sensitivities in a subgroup of patients with idiopathic autism.

\section{CONFLICT OF INTEREST}

JAS served as a consultant to Takeda, Roche and Lilly. MJB reports research grant funding and speaker's bureau support from Otsuka. The remaining authors declare no conflict of interest.

\section{ACKNOWLEDGMENTS}

We would like to thank Rachel Greene, Savanna Sablich and Melanie Soilleux for aid in data collection. This study was supported by NIMH/NICHD grant U54 HD082008-01 (Huber/JAS). The sponsor had no further role in the research plan development, analysis or reporting of results.

\section{REFERENCES}

1 Chonchaiya W, Schneider A, Hagerman RJ. Fragile X: a family of disorders. Adv Pediatr 2009; 56: 165-186.

2 Hagerman PJ. The fragile X prevalence paradox. J Med Genet 2008; 45: 498-499.

3 Matsuzaki J, Kagitani-Shimono K, Goto T, Sanefuji W, Yamamoto T, Sakai S et al. Differential responses of primary auditory cortex in autistic spectrum disorder with auditory hypersensitivity. Neuroreport 2012; 23: 113-118.

4 Hall SS, Walter E, Sherman E, Hoeft F, Reiss AL. The neural basis of auditory temporal discrimination in girls with fragile X syndrome. J Neurodev Disord 2009; 1: 91-99.
5 Gibson JR, Bartley AF, Hays SA, Huber KM. Imbalance of neocortical excitation and inhibition and altered UP states reflect network hyperexcitability in the mouse model of fragile X syndrome. J Neurophysiol 2008; 100: 2615-2626.

6 Rotschafer S, Razak K. Altered auditory processing in a mouse model of fragile $X$ syndrome. Brain Res 2013; 1506: 12-24.

7 Barnes SA, Pinto-Duarte A, Kappe A, Zembrzycki A, Metzler A, Mukamel EA et al. Disruption of mGluR5 in parvalbumin-positive interneurons induces core features of neurodevelopmental disorders. Mol Psychiatry 2015; 20: 1161-1172.

8 Paluszkiewicz SM, Olmos-Serrano JL, Corbin JG. Huntsman MM. Impaired inhibitory control of cortical synchronization in fragile X syndrome. J Neurophysiol 2011; 106: 2264-2272.

9 Womelsdorf T, Lima B, Vinck M, Oostenveld R, Singer W, Neuenschwander S et al. Orientation selectivity and noise correlation in awake monkey area $\mathrm{V} 1$ are modulated by the gamma cycle. Proc Natl Acad Sci USA 2012; 109: 4302-4307.

10 Devitt NM, Gallagher L, Reilly RB. Autism spectrum disorder (ASD) and fragile X syndrome (FXS): two overlapping disorders reviewed through electroencephalography-what can be interpreted from the available Information? Brain Sci 2015; 5: 92-117.

11 Castren M, Paakkonen A, Tarkka IM, Ryynanen M, Partanen J. Augmentation of auditory N1 in children with fragile X syndrome. Brain Topogr 2003; 15: 165-171.

12 Van der Molen MJ, Van der Molen MW, Ridderinkhof KR, Hamel BC, Curfs LM, Ramakers GJ. Auditory change detection in fragile $X$ syndrome males: a brain potential study. Clin Neurophysiol 2012; 123: 1309-1318.

13 Knoth IS, Vannasing P, Major P, Michaud JL, Lippe S. Alterations of visual and auditory evoked potentials in fragile X syndrome. Int J Dev Neurosci 2014; 36: 90-97.

14 Budd TW, Barry RJ, Gordon E, Rennie C, Michie PT. Decrement of the N1 auditory event-related potential with stimulus repetition: habituation vs. refractoriness. Int J Psychophysiol 1998; 31: 51-68.

15 Wang P, Knosche TR. A realistic neural mass model of the cortex with laminarspecific connections and synaptic plasticity - evaluation with auditory habituation. PLoS One 2013; 8: e77876.

16 Kisley MA, Noecker TL, Guinther PM. Comparison of sensory gating to mismatch negativity and self-reported perceptual phenomena in healthy adults. Psychophysiology 2004; 41: 604-612.

17 Greife CL. Sensory gating, habituation, and orientation of P50 and N100 eventrelated potential (ERP) components in neurologically typical adults and links to sensory behaviors. MS thesis. Colorado State University: Fort Collins, Colorado, 2013.

18 Schneider A, Leigh MJ, Adams P, Nanakul R, Chechi T, Olichney J et al. Electrocortical changes associated with minocycline treatment in fragile $\mathrm{X}$ syndrome. $J$ Psychopharmacol 2013; 27: 956-963.

19 Clearwater JM, Kerr CC, Rennie CJ, Robinson PA. Neural mechanisms of ERP change: combining insights from electrophysiology and mathematical modeling. J Integr Neurosci 2008; 7: 529-550.

20 Cowan N. Sensory memory and its role in information processing. Electroencephalogr Clin Neurophysiol Suppl 1995; 44: 21-31.

21 Naatanen R, Teder W, Alho K, Lavikainen J. Auditory attention and selective input modulation: a topographical ERP study. Neuroreport 1992; 3: 493-496.

22 Adler LE, Pachtman E, Franks RD, Pecevich M, Waldo MC, Freedman R. Neurophysiological evidence for a defect in neuronal mechanisms involved in sensory gating in schizophrenia. Biol Psychiatry 1982; 17: 639-654.

23 Dolu N, Suer C, Ozesmi C. A comparison of the different interpair intervals in the conditioning-testing P50 paradigms. Int J Psychophysiol 2001; 41: 265-270.

24 Hamm JP, Ethridge LE, Boutros NN, Keshavan MS, Sweeney JA, Pearlson GD et al. Diagnostic specificity and familiality of early versus late evoked potentials to auditory paired stimuli across the schizophrenia-bipolar psychosis spectrum. Psychophysiology 2014; 51: 348-357.

25 Brown C, Tollefson N, Dunn W, Cromwell R, Filion D. The Adult Sensory Profile: measuring patterns of sensory processing. Am J Occup Ther 2001; 55: 75-82.

26 Roid GH. Stanford-Binet Intelligence Scales, 5th edn (SB:V). Riverside Publishing: Itasca, IL, USA, 2003.

27 Wechsler D. Wechsler Abbreviated Scale of Intelligence (WASI). Harcourt Assessment: San Antonio, TX, USA, 1999.

28 Chatrian GE. Ten percent electrode system for topographic studies of spontaneous and evoked EEG activity. Am J EEG Technol 1985; 25: 83-92.

29 Delorme A, Makeig S. EEGLAB: an open source toolbox for analysis of single-trial EEG dynamics including independent component analysis. J Neurosci Methods 2004; 134: 9-21.

30 Ethridge LE, Hamm JP, Shapiro JR, Summerfelt AT, Keedy SK, Stevens MC et al. Neural activations during auditory oddball processing discriminating schizophrenia and psychotic bipolar disorder. Biol Psychiatry 2012; 72: 766-774.

31 Ethridge LE, Hamm JP, Pearlson GD, Tamminga CA, Sweeney JA, Keshavan MS et al. Event-related potential and time-frequency endophenotypes for schizophrenia and psychotic bipolar disorder. Biol Psychiatry 2015; 77: 127-136.

32 Busch NA, VanRullen R. Spontaneous EEG oscillations reveal periodic sampling of visual attention. Proc Natl Acad Sci USA 2010; 107: 16048-16053. 
33 Hamm JP, Ethridge LE, Shapiro JR, Stevens MC, Boutros NN, Summerfelt AT et al. Spatiotemporal and frequency domain analysis of auditory paired stimuli processing in schizophrenia and bipolar disorder with psychosis. Psychophysiology 2012; 49: 522-530.

34 Rosburg T, Zimmerer K, Huonker R. Short-term habituation of auditory evoked potential and neuromagnetic field components in dependence of the interstimulus interval. Exp Brain Res 2010; 205: 559-570.

35 Fruhstorfer $\mathrm{H}$, Jarvilehto $\mathrm{T}$, Soveri $\mathrm{P}$. Short-term habituation and dishabituation of the sensory evoked response in man. Acta Physiol Scand 1969; 76: 14A-15A.

36 Frankland PW, Wang Y, Rosner B, Shimizu T, Balleine BW, Dykens EM et al. Sensorimotor gating abnormalities in young males with fragile $\mathrm{X}$ syndrome and Fmr1-knockout mice. Mol Psychiatry 2004; 9: 417-425.

37 Yuhas J, Cordeiro L, Tassone F, Ballinger E, Schneider A, Long JM et al. Brief report: sensorimotor gating in idiopathic autism and autism associated with fragile $X$ syndrome. J Autism Dev Disord 2011; 41: 248-253.

38 Hessl D, Berry-Kravis E, Cordeiro L, Yuhas J, Ornitz EM, Campbell A et al. Prepulse inhibition in fragile $X$ syndrome: feasibility, reliability, and implications for treatment. Am J Med Genet B Neuropsychiatr Genet 2009; 150B: 545-553.

39 Miller LJ, McIntosh DN, McGrath J, Shyu V, Lampe M, Taylor AK et al. Electrodermal responses to sensory stimuli in individuals with fragile $X$ syndrome: a preliminary report. Am J Med Genet 1999; 83: 268-279.

40 Moratti S, Clementz BA, Gao Y, Ortiz T, Keil A. Neural mechanisms of evoked oscillations: stability and interaction with transient events. Hum Brain Mapp 2007; 28: $1318-1333$.

41 Patel SH, Azzam PN. Characterization of N200 and P300: selected studies of the Event-Related Potential. Int J Med Sci 2005; 2: 147-154.

42 Pantev C, Makeig S, Hoke M, Galambos R, Hampson S, Gallen C. Human auditory evoked gamma-band magnetic fields. Proc Natl Acad Sci USA 1991; 88: 8996-9000.
43 Vianney-Rodrigues P, lancu OD, Welsh JP. Gamma oscillations in the auditory cortex of awake rats. Eur J Neurosci 2011; 33: 119-129.

44 Bear MF, Huber KM, Warren ST. The mGluR theory of fragile X mental retardation. Trends Neurosci 2004; 27: 370-377.

45 Barnet $A B$, Ohlrich ES, Shanks BL. EEG evoked responses to repetitive auditory stimulation in normal and Down's syndrome infants. Dev Med Child Neurol 1971; 13: $321-329$.

46 Schafer EW, Peeke HV. Down syndrome individuals fail to habituate cortical evoked potentials. Am J Ment Defic 1982; 87: 332-337.

47 Babiloni C, Albertini G, Onorati P, Muratori C, Buffo P, Condoluci C et al. Cortical sources of EEG rhythms are abnormal in down syndrome. Clin Neurophysiol 2010; 121: $1205-1212$.

48 Politoff AL, Stadter RP, Monson N, Hass P, Cognition-related EEG. abnormalities in nondemented Down syndrome subjects. Dementia 1996; 7: 69-75.

49 Dansie LE, Phommahaxay K, Okusanya AG, Uwadia J, Huang M, Rotschafer SE et al. Long-lasting effects of minocycline on behavior in young but not adult Fragile X mice. Neuroscience 2013; 246: 186-198.

50 Lovelace JW, Wen TH, Reinhard S, Hsu MS, Sidhu H, Ethell IM et al. Matrix metalloproteinase-9 deletion rescues auditory evoked potential habituation deficit in a mouse model of Fragile X Syndrome. Neurobiol Dis 2016; 89: 126-135.

This work is licensed under a Creative Commons Attribution 4.0 International License. The images or other third party material in this article are included in the article's Creative Commons license, unless indicated otherwise in the credit line; if the material is not included under the Creative Commons license, users will need to obtain permission from the license holder to reproduce the material. To view a copy of this license, visit http://creativecommons.org/licenses/ by/4.0/

Supplementary Information accompanies the paper on the Translational Psychiatry website (http://www.nature.com/tp) 